\title{
Associations between perceived participation outside home and types of places visited among persons living with and without dementia: risks perception and socio-demographic aspects.
}

\section{Isabel Margot-Cattin ( $\boldsymbol{Q}$ isabel.margot@hetsl.ch )}

UNiversity of Applied Sciences and Arts Western Switzerland (HES-SO)

André Berchtold

University of Lausanne

Sophie Gaber

Karolinska Institute

Nicolas Kühne

UNiversity of Applied Sciences and Arts Western Switzerland (HES-SO)

Louise Nygard

Karolinska Institute

Camilla Malinowsky

Karolinska Institute

\section{Research Article}

Keywords: Participation outside home, Dementia, Risks perception, Places visited

Posted Date: February 9th, 2022

DOl: https://doi.org/10.21203/rs.3.rs-1320370/v1

License: (c) (i) This work is licensed under a Creative Commons Attribution 4.0 International License.

Read Full License 


\section{Abstract}

\section{Introduction:}

Participation outside home has recently been given attention with a focus on places visited for persons living with and without dementia. Multiple factors like socio-demographic aspects and risk perception have been shown to influence the perception of participation outside home.

\section{Aim and methods:}

This cross-sectional, explorative study aims to explore how places visited, socio-demographic aspects and risk perception are associated with the perception of participation outside home for a sample of 70 persons living with and without dementia in Switzerland. First, we investigated whether the number of places visited was correlated with the perception of participation outside home; then we added sociodemographic and risk perception factors with a bivariate analysis; and searched for a model using multinomial logistic regressions.

\section{Results:}

The number of places visited was associated with the perception of participation outside home, but only for the group of persons living without dementia $(p=.043)$. Risks of falling $(p=.014)$ and of getting lost $(p=.037)$ were associated for the group of persons living with dementia only. Being able to visit places for recreational and physical activities $(p=.005)$ was associated for the group of persons living without dementia.

\section{Discussion and conclusion:}

The results of the exploration of multiple factors and search for a model, highlights the complexity of participation outside home as a construct. Visiting multiple and various types of places seem to play a role in participation outside home but would need to be further investigated.

\section{Introduction}

Dementia is a condition which include progressive memory impairment and loss of other cognitive functions $(1,2)$. While cognitive decline in dementia has been widely and thoroughly examined $(3,4)$, participation and engagement in everyday activities have been less studied $(5,6)$; and participation outside home even less $(7,8)$.

These last few years have seen an increasing volume of literature on out-of-home participation, including a focus on a specifically delimited place, the neighbourhood as being of importance for persons living with dementia (9-11). A series of studies have lately been conducted on participation outside home focusing on places visited by persons living with submitted, in Canada, Sweden, Switzerland and the United Kingdom. The results of these studies have shown that persons living with dementia are likely to 
experience not just a global decrease of their participation outside home, but rather a shift from visiting social and cultural places to places used for medical and self-care (12) They have also been shown to experience significantly more abandonment of places than their peers without dementia, showed from comparisons of number of places visited in the past to places presently visited (13). The studies mentioned above are based on the assumption that visiting places is partly an expression of participation outside home (14-16). However, knowledge of how the number of places visited would be associated with older adults' perception of their own participation is not yet known.

Additionally, socio-demographic factors have been highlighted as influencing participation outside home and how it is perceived by persons living with dementia, showing that a multitude of factors may have an impact (17). These include intrapersonal factors like age, gender and education level; interpersonal factors like living alone or with someone (18); and environmental factors like living in a rural or urban context (19). Availability of amenities, transportation, and driving issues have been found to have an impact on participation outside home $(20,21)$.

Additional limiting factors for out-of-home participation include old age, female gender, low socioeconomic status, social deprivation of the living environment, comorbidity, lifestyle, lack of motivation, weak social network and limited social activities $(22,23)$. Being a driver $(24)$ or losing access to a car (21) are factors or events that also have an impact on participation outside home. Having a dementia adds a factor (7) and underscores the complexity of understanding what influences participation outside home and its perception.

Furthermore, how risks are constructed and perceived have been identified as playing a role in participation outside home and dementia care $(25,26)$. Research on risks has mostly been on health consequences, safety issues and prevention of the event identified as risk (27). As such, persons living with dementia may experience family, friends and service providers framing risks as "unwanted", "undesirable" or "to be avoided". This construction of risks is based on the importance given to safety in dementia care. As a result, older adults with dementia have reported reducing their involvement in activities outside home and confining themselves to home as a way of increasing safety $(28,29)$. This construction of risks focuses on a depiction of "risk" as negative or dangerous, stressing the potential loss; instead of a more nuanced concept that can bring "good" or "gains" to the person living with dementia $(30,31)$. There are positive aspects of risk taking, like expressing one's individuality, independence and control over one's life; which could reframe activities outside home that appear hazardous into ones perceived as positive and identity enhancing $(32,33)$. A more nuanced approach has been considering risks for persons living with dementia as potentially experience a sense of independence, freedom, and control.

Getting lost is a commonly perceived risk by persons living with dementia and their families. It is often associated with wandering behaviour and is often used as a reason to limit participation outside home $(34,35)$. Falling is another commonly perceived risk, but by both persons living with and without dementia, and fear of falling is an aggravating factor. Research suggests that approximately half of all 
falls occur in the street or in places outside home (36). Feeling stressed or embarrassed are also identified as a risk by the persons living with dementia (37). Despite this, little research has examined how the way persons perceive these risks might be associated with their perceived participation outside home.

A study on risk construction and perception conducted in Switzerland with triads of persons living with dementia, their significant others and home-health professionals, has shown that risks need to be understood as dynamic, co-constructed (38). Coping strategies are often used by persons living with dementia to manage situations fraught with risks, like staying at home and withdrawing from out-ofhome activities, asking for help to be driven to appointments, relying on significant others to go out; and for some persons simply being aware of risks, tolerating them when going out, and even experiencing them as challenges to be overcome (39). How risks are seen, constructed, and experienced in the situation of persons living with dementia may shape how they participate outside home, thus underscoring the need to examine their perceptions.

In summary, the number of places visited might have an influence on how older people perceive their participation outside home. As participation outside home is a complex, dynamic, and multi-faceted phenomenon, other factors than visiting places may shape how persons living with and without dementia experience and perceive it. Such additional factors are socio-demographic variables and perceived risks. To our knowledge, these factors have not yet been investigated in relation to the perception of participation outside home.

The aim of this study is to explore how these factors are associated with the perception of participation outside home for a sample of persons living with and without dementia in Switzerland. The research questions this study addresses are:

1) What associations exist between the number of places visited and the perception of their participation outside home for a sample of persons living with and without dementia?

2) What factors, e.g., amount and types of places visited, socio-demographic variables and perceived risks, might be associated with the perception of participation outside home?

\section{Methods}

\section{Design and setting}

This cross-sectional, explorative study reports on research undertaken within the "Life outside home for people with dementia" (OUTDEM) setting as part of a larger project in leadership of Karolinska Institutet in Sweden. Standardized questionnaires were used in interviews with participants with and without dementia living in the French-speaking region of Switzerland, in both rural and urban regions. 


\section{Participants and recruitment}

All participants $(n=70)$ were community-dwelling older adults $(65+)$. Recruitment started in December 2015 and ended in May 2017. Participants with dementia $(n=35)$ were recruited through memory clinics, day hospitals, and the Swiss Alzheimer's association. Diagnosis of dementia was established by physicians at memory clinics. Participants in the comparison group $(n=35)$ were recruited through senior associations and advertisements in grocery stores. The comparison group without known dementia $(n=35)$ was aimed to match, but not paired with the dementia group regarding age, gender, living areas and settings, and education level; thus, recruitment strategies for the comparison group targeted specific regions, age groups, or living areas, for example, to bring the distribution of the comparison group closer to the dementia group on those variables.

An approximate required sample size was calculated based on the difference of the total number of places visited between the 26 older adults and the five persons living with dementia who took part in the study presenting ACT-OUT development (40). The same approximate sample size of groups of 35 were used in similar studies $(13,41)$. No formal power calculation was conducted due to the exploratory design. Findings of this study might be used with former ones to generate power calculations for future research using ACT-OUT.

\section{Data collection procedures}

The interviews were conducted by two registered occupational therapists of which one was the first author. Both interviewers had prior knowledge of using ACT-OUT and had harmonized the way they conducted the interviews (42). The interview comprised of three standardized questionnaires, performed in this order: (i) the Participation in ACTivities and Places OUTside Home Questionnaire (ACT-OUT) (40); (ii) the Montreal Cognitive Assessment (MoCA) (43); (iii) socio-demographic questions. Written and verbal informed consent was obtained from each participant prior to data collection. The process consent method proposed by Dewing (2002) (44) was used in this study, because it is person-centred; and it enables researchers to include consent communicated through behaviour and non-verbal means by the person with dementia. Thus, following Dewing (2007) (45), an ongoing consent monitoring was implemented throughout the whole data collection to ensure no stress or burden from participating in the project occurred (46). To mitigate against fatigue and potential burden, interviews occurred in the participant's home and were adapted to each participant e.g. inviting a significant other for emotional support or spreading the sessions, of no more than two hours. An ethical authorization (protocol 452/15) was obtained from the "Commission cantonale d'éthique de la recherche sur l'être humain (CER-VD)" in Lausanne, Switzerland.

\section{Data analysis: questionnaires and variables}


ACT-OUT has three parts. Part I includes a list of 25 pre-determined types of places, grouped into four domains: A, consumer, administrative, and self-care places $(n=7)$; B, places for medical care $(n=5)$; C, social, cultural, and spiritual places $(n=6)$; and D, places for recreational and physical activities $(n=7)$. Part I asks questions whether respondents visit these places in the past, present, and future. Part II poses detailed questions about factors potentially influencing participation in places retained and abandoned, like activities performed, transportation means, accompanying persons, risk perception, and familiarity. Part III consists of general questions about perceived out-of-home participation, life satisfaction, and attitudes towards risk-taking and stress factors.

The Montreal Cognitive Assessment (MoCA) (43) was used as a comprehensive screening tool for assessing and describing the level of cognitive functioning for both groups. It is made of sections focusing on diverse cognitive functions (memory, time and space orientation, visual perception, for example). The total score reflects the cognitive level of participants.

In this study, data from ACT-OUT (Part I and III), MoCA total score and socio-demographic questions were used.

\section{Dependant variable: perceived participation}

The dependent variable is the perceived out-of-home participation question asked in Part III of ACT-OUT (How do you perceive your participation in all situations outside home to be?) to which participants responded using a Likert scale ( $4=$ I participate as I wish; $3=$ I participate almost as I wish; 2 = I rather do not participate as I wish; 1 = I do not participate as I wish). Of the four levels only one participant with dementia responded do not participate as I wish, thus allowing the aggregation of levels 1 and 2.

\section{Measures: independent variables}

The 15 independent variables were derived from the ACT-OUT questionnaire (Part I: number of places visited $(n=5)$ and Part III: risks $(n=4))$ and socio-demographic questions $(n=6)$. The five variables from part I are first the total number of places visited $(n=25)$, which is the sum of the number of places in all four domains, the following four are the number of places visited in each of the four domains $A /$ consumer, administrative, and self-care places $(n=7), B /$ places for medical care $(n=5), C /$ social, cultural, and spiritual places $(n=6)$, and $D /$ places for recreational and physical activities $(n=7)$. The categorization into domains allows for the type of places to be highlighted.

The independent variables from the socio-demographic questionnaire are: (i) age (years); (ii) gender (male or female); (iii) living situation (alone or with someone); (iv) education level, adapted into three levels from The International Standard Classification of Education (ISCED 2011) (47) (primary/secondary school, apprenticeship and higher education degree); (v) rural/urban, adapted to the context as village, 
small town and city; (vi) use of car, (access to a car as self-driving, someone else drives, and no use of car).

In ACT-OUT Part III, four risks influencing out-of-home participation, identified in the literature about people with dementia $(37,39)$ are questioned with a four level Likert-type rating scale about the concern that respondents perceive (very concerned; concerned; unconcerned; very unconcerned). The four types of perceived risks were (i) falling; (ii) getting lost; (iii) feeling stressed; (iv) feeling embarrassed. As no participants responded very concerned on any of the risks, these variables were transformed into a threelevel Likert-type rating scale.

\section{Statistical analyses}

First, we computed descriptive statistics for all variables, and we systematically tested the difference between the dementia and comparison groups using the Fisher exact test for categorical variables, and the t-test for continuous variables. To explore the associations between total number of places and the perception of out-of-home participation, Spearman correlations coefficients were evaluated for the dementia group and the comparison group separately, as well as for the full group of 70 participants. The strength of association was classified using Cohen's guidelines for social sciences: .1-.3 (small); .3-.5 (medium); and .5-1.0 (large) (48). For testing the differences between the total number of places by the level of perceived out-of-home participation, we used the Mann-Whitney $U$ test for the comparison group (two levels of perceived participation) and the Kruskal-Wallis test for the dementia group (three levels of perceived participation). Notice that we had to use non-parametric tests because of the non-normality of data and of the small sample sizes.

Second, to explore the associations between each of our independent variables and perceived out-ofhome participation, bivariate regressions were run. As the group variable was significant $(p<.001)$ in the full sample analyses, it was decided to run the regressions for the dementia and comparison groups separately. We used logistic regressions for the comparison group, since the dependent variables had two categories, and multinomial regressions for the dementia group, since the dependent variable had three categories. At this point, we selected all independent variables significant at the bivariate level and we entered them together into multivariate regressions.

For all regressions, the highest level of perceived participation was used as the reference category. The type I error was set to .05 for all analyses. Results are given as relative risk ratios (RRR) with $95 \%$ confidence intervals for the multinomial regressions, and as odds ratio (OR) with $95 \%$ confidence intervals for the logistic regressions. Analyses were undertaken in the Statistical Package for Social Sciences (SPSS) computer software, version 25.

\section{Results}


Descriptive statistics for the sample are presented in Table 1 for both the dementia and comparison group respectively. The groups significantly differed regarding the dependent variable, the number of places visited, the MoCA score and all the risks but falling down. The groups did not significantly differ for all socio-demographic variables but the access to a car.

\section{Table 1}

Description of the outcome (dependent) variable and independent variables. For the comparison test, we used the Fisher exact test for categorical variables, and the t-test for continuous variables. 


\begin{tabular}{|c|c|c|c|c|c|}
\hline \multirow[t]{2}{*}{ Variables } & Dementia & Comparison & \multirow{2}{*}{$\begin{array}{l}\mathrm{Cl} 95 \% \text { of } \\
\text { mean } \\
\text { difference }\end{array}$} & \multirow{2}{*}{$\begin{array}{l}\text { Coefficient for the } \\
\text { difference between } \\
\text { groups }\end{array}$} & \multirow{2}{*}{$\begin{array}{l}p- \\
\text { value }\end{array}$} \\
\hline & $n=35$ & $n=35$ & & & \\
\hline $\begin{array}{l}\text { Outcome: Participation } \\
\text { (ACT-OUT, Part 3) }\end{array}$ & & & & 18.740 & $<.001$ \\
\hline Participate only a little & 12 & 0 & & & \\
\hline Do participate & 8 & 6 & & & \\
\hline $\begin{array}{l}\text { Participate as much as } \\
\text { wanted }\end{array}$ & 15 & 29 & & & \\
\hline \multicolumn{6}{|c|}{ Number of visited places by domain (ACT-OUT) } \\
\hline $\begin{array}{l}\text { Nb of currently visited } \\
\text { places (ACT-OUT, max = } \\
25 \text { ) }\end{array}$ & & & & 3.893 & $<.001$ \\
\hline Mean (SD) & $\begin{array}{l}15.83 \\
(3.34)\end{array}$ & $18.91(3.28)$ & $1.50 ; 4.66$ & & \\
\hline $\begin{array}{l}\text { Nb visited places - } \\
\text { Domain A } \\
(\max =7)\end{array}$ & & & & 4.622 & $<.001$ \\
\hline Mean (SD) & $\begin{array}{l}4.43 \\
(2.11)\end{array}$ & $6.31(1.15)$ & $1.07 ; 2.70$ & & \\
\hline $\begin{array}{l}\text { Nb visited places - } \\
\text { Domain B } \\
(\max =5)\end{array}$ & & & & -2.653 & .010 \\
\hline Mean (SD) & $\begin{array}{l}3.26 \\
(0.98)\end{array}$ & $2.71(0.71)$ & $-.95 ;-.13$ & & \\
\hline $\begin{array}{l}\text { Nb visited places - } \\
\text { Domain C } \\
(\max =6)\end{array}$ & & & & 3.144 & .002 \\
\hline Mean (SD) & $\begin{array}{l}3.71 \\
(1.25)\end{array}$ & $4.66(1.25)$ & $.34 ; 1.54$ & & \\
\hline $\begin{array}{l}\text { Nb visited places - } \\
\text { Domain D } \\
(\max =7)\end{array}$ & & & & 2.661 & .010 \\
\hline Mean (SD) & $\begin{array}{l}4.11 \\
(1.18)\end{array}$ & $5.03(1.65)$ & $.23 ; 1.60$ & & \\
\hline \multicolumn{6}{|c|}{ Characteristics of the participants (MoCA and socio-demographic questionnaire) } \\
\hline MoCA & & & & 8.195 & $<.001$ \\
\hline
\end{tabular}




$\begin{array}{lrrr}\text { Mean (SD) } & 17.74 & 26.09(2.07) & 6.31 ; \\ & (5.56) & & 10.37\end{array}$

\section{Age}

Mean (SD)

Gender

Male

Female

Education

Mandatory school

Apprenticeship

Higher education degree

Living arrangement

Alone

Living with someone

Setting

Rural

Small town

City

Access to using a car

Not a car user

Car user (self-driving)

Car user (someone else drives)

Perceived risks (ACT-OUT)

Getting lost

77.66

(8.35)

$77.86(7.72) \quad-3.63 ; 4.03$

.952

.333

16

19

12

23

110

.950

$9 \quad 10$

$17 \quad 17$

9

8

0

7

8




\begin{tabular}{|c|c|c|c|c|}
\hline Unconcerned & 8 & 11 & & \\
\hline Very unconcerned & 20 & 21 & & \\
\hline Being stressed & & & 9.762 & .008 \\
\hline Concerned & 10 & 2 & & \\
\hline Unconcerned & 10 & 6 & & \\
\hline Very unconcerned & 15 & 27 & & \\
\hline Being embarrassed & & & 11.427 & .003 \\
\hline Concerned & 11 & 2 & & \\
\hline Unconcerned & 7 & 3 & & \\
\hline Very unconcerned & 17 & 30 & & \\
\hline
\end{tabular}

a) Association between the number of places visited and perceived participation

The full group showed a significant correlation $(p=.036)$ between perceived participation and total number of places visited; the strength of the association was small $\left(r_{s}=.251\right)$ and positive, meaning that the more places persons visit, the better they rated their participation. The comparison group showed a significant correlation ( $p=.043)$; the strength was medium $\left(r_{s}=.344\right)$ and positive. In the dementia group, the correlation was not significant. Figure 1 illustrates the mean number of places visited in relation to the perceived participation rated by each group. When looking at the mean of the number of places visited by participants based on their self-reported level of participation outside home, it shows that the comparison group had a significant decrease of the mean of places visited ( $p=.044)$, compared to the dementia group which shows non-significant fluctuations (See Figure 1).

b) Exploration of associations between perceived out-of-home participation and multiple variables

Although the education variable has been identified as a factor influencing participation outside the home, it proved to be problematic in our samples, since there were not enough participants in each category to calculate relative risk ratios for all three groups; and so, we decided to remove it from the bivariate regressions. Most of the variables proved to be not significant at the bivariate level, for either group samples.

For the group of participants living with dementia, results are presented in Table 2. Only the risk variables of getting lost $(p=.037)$ and falling $(p=.014)$ were significantly associated in the bivariate multinomial regressions and were included in the multivariate regression. In the multivariate regression, no independent variable was significant. Both risks are reported as hindering their perceived participation outside home.

\section{Table 2}


Bivariate and multivariate multinomial regressions for the level of participation for the dementia group, using "participate as I wish" as the reference category 
Group living with dementia: Bivariate (each variable treated separatedly)

Independent variable Participate only a little Do participate (3)

(2)

$\begin{array}{llllll}\mathrm{RRR} & \mathrm{Cl} & p \text { - } & \mathrm{RRR} & \mathrm{Cl} & p \text { - } \\ & 95 \% & \text { value }\end{array} \quad \begin{array}{ll}95 \% & \text { value }\end{array}$

$\begin{array}{ll}\text { Nagelkerke } & p- \\ \text { value }\end{array}$ $\mathrm{R} 2$

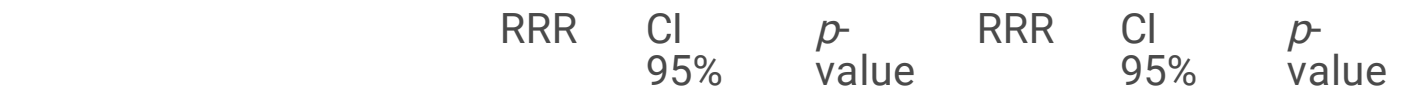

Risks

Getting lost (ref: very

.287

.037

unconcerned (4))

$\begin{array}{lcccccc}\text { Concerned (2) } & 4.20 & .59- & .153 & 2.10 & .25- & .494 \\ & & 30.09 & & & 17.59 & \\ \text { Unconcerned (3) } & .23 & \begin{array}{ll}.02- \\ 2.59\end{array} & .236 & \text { (a) } & \text { (a) } & \text { (a) }\end{array}$

Falling down (ref: very

unconcerned (4))

$\begin{array}{lllllll}\text { Concerned (2) } & \text { (a) } & \text { (a) } & \text { (a) } & \text { (a) } & \text { (a) } & \text { (a) } \\ \text { Unconcerned (3) } & 1.38 & .18- & .760 & 1.10 & .15- & .926 \\ & & 10.65 & & & 8.13 & \end{array}$

Stress (ref: very

unconcerned (4))

.033

.904

Concerned (2)

$.84 \quad .13-$

$.852 \quad .93$

$.11-$

7.82

.949

.340

.014

Unconcerned (3)

$1.87 \quad .28-$

12.31

.517

2.33

$.29-$

18.97

.428

Embarrassment (ref:

very unconcerned (4))

\begin{tabular}{|c|c|c|c|c|c|c|}
\hline Concerned (2) & 1.94 & $\begin{array}{l}.32- \\
11.76\end{array}$ & .469 & 1.75 & $\begin{array}{l}.23- \\
13.16\end{array}$ & .587 \\
\hline Unconcerned (3) & .23 & $\begin{array}{l}.02- \\
2.59\end{array}$ & .236 & .35 & $\begin{array}{l}.03- \\
4.15\end{array}$ & .406 \\
\hline
\end{tabular}

Socio-demographic

Age

1.00

.91- $\quad .996 \quad .99$

$.90-$

1.10

.111

.462

Gender (ref: female

(2))

.48

1.09

(1.

Living situation (ref:

with someone (2))

.50

$\begin{array}{lll}.10- & .346 & .22\end{array}$

.03-

1.50

.921

.000

.994

Access to car (ref:

someone else drives

$.07-$

3.36

.122

.086

.250 
(2))

\begin{tabular}{lllllll} 
Not a car user (0) & 1.12 & $.19-$ & .899 & .56 & $.08-$ & .570 \\
& & 6.41 & & & 4.14 & \\
$\begin{array}{l}\text { Car user - self-driving } \\
(1)\end{array}$ & 1.40 & $.19-$ & .738 & .47 & $.04-$ & .467 \\
\hline
\end{tabular}

Setting (ref: big city

(2))

$\begin{array}{lcccccc}\text { Rural (0) } & 1.00 & \begin{array}{c}.13- \\ 7.57\end{array} & 1.00 & \text { (a) } & \text { (a) } & \text { (a) } \\ \text { Small town (1) } & .57 & .08- & .587 & \text { (a) } & \text { (a) } & \text { (a) } \\ & & 4.29 & & & & \end{array}$

Places visited

Total number of

places visited

$\begin{array}{lllllll}1.08 & .85- & .510 & .95 & .74- & .710 & .029\end{array}$

.635

$\begin{array}{lllllllll}\text { Total places visited in } & 1.00 & .70- & 1.00 & 1.11 & .72- & .647 & .008 & .881\end{array}$

A

$\begin{array}{llllllll}\text { Total places visited in } & 1.49 & .67- & .334 & .99 & .40- & .984 & .037\end{array}$

B

$3.33 \quad 2.47$

$\begin{array}{lllllllll}\text { Total places visited in } & .88 & .46- & .710 & .49 & .22- & .079 & .115 & .155 \\ \mathrm{C} & & 1.71 & & & 1.09 & & & \end{array}$

C

$\begin{array}{lllllllll}\text { Total places visited in } & 1.87 & .83- & .133 & .67 & .30- & .316 & .167 & \\ \text { D } & & 4.22 & & & 1.48 & & & \end{array}$

Group living with dementia : Multivariate (Nagelkerke R2 .468, $p=.017$ )

Independent variable Participate only a little Do participate (3) p-value (2)

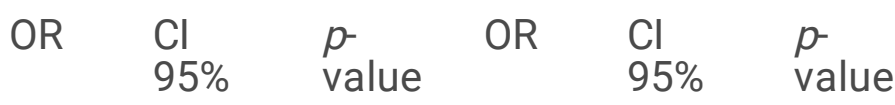

Getting lost (ref: very

unconcerned (4))

$\begin{array}{lcccccc}\text { Concerned (2) } & 1.71 & \begin{array}{c}.15- \\ 19.19\end{array} & .663 & 1.70 & .18- & .644 \\ & & & & 16.46 & \\ \text { Unconcerned (3) } & .35 & \begin{array}{c}.03- \\ 4.20\end{array} & .406 & \text { (a) } & \text { (a) } & \text { (a) }\end{array}$

Falling down (ref: very unconcerned (4))
Concerned (2)
(a) (a)
(a)
(a)
(a)
(a)

Unconcerned (3)

$\begin{array}{llllll}1.24 & .14- & .848 & .98 & .10- & .988 \\ & 11.06 & & & 9.58 & \end{array}$


(a )Not enough participants in this category to get results.

Table 3 shows the results from the set of bivariate multinomial regressions for the comparison group sample. The results for the risk variables show that most participants in the comparison group perceived risks as little related to their perception of participation outside home. Only the total number of places visited in $D$ domain $(p=.005)$ was significantly associated. Since only one predictor was significant for the comparison group at the bivariate level, no additional regression was required.

\section{Table 3}

Bivariate logistic regressions for the level of participation for the comparison group, using "participate as much as I want" as the reference category, each independent variable treated separately 


\section{Comparison group}

Independent variable

Do participate (3)

Nagelkerke $p$-value

OR $\mathrm{Cl} 95 \% \quad p$-value R2

Risks

Getting lost (ref: very unconcerned (4))

(a)

(a)

Unconcerned (3)

(a) (a) (a)

Falling down (ref: very unconcerned (4))

Concerned (2)

(a) (a) (a)

Unconcerned (3)

$5.43 \quad .81-36.51 \quad .082$

Stress (ref: very unconcerned (4))

.218

.086

Concerned (2)

(a) (a) (a)

Unconcerned (3)

$\begin{array}{lll}8.00 & 1.08-59.14 \quad .042\end{array}$

Embarrassment (ref: very unconcerned (4))

Concerned (2)

(a) (a) (a)

Unconcerned (3)

$\begin{array}{lll}2.50 & .19-33.17 \quad .487\end{array}$

Socio-demographic

\begin{tabular}{|lccccc|}
\hline Age & 1.08 & $.96-1.23$ & .212 & .080 & .191 \\
\hline Gender (ref: female (2)) & 2.22 & $.37-13.22$ & .380 & .036 & .383 \\
\hline Living situation (ref: with someone (2)) & .25 & $.03-2.38$ & .246 & .084 & .178 \\
\hline Access to car (ref: someone else drives (2)) & & & & .003 & .971 \\
\hline Not a car user (0) & .83 & $.04-16.99$ & .906 & & .666 \\
\hline Car user - self-driving (1) & 1.11 & $.10-12.31$ & .932 & & .038 \\
\hline Setting (ref: big city (2)) & & & & & \\
\hline Rural (0) & 2.73 & $.24-30.66$ & .416 & .164 \\
\hline Small town (1) & 2.50 & $.19-32.80$ & .485 & & .493 \\
\hline Places visited & & & & .090 & .252 \\
\hline Total number of places visited & .83 & $.64-1.08$ & .172 & .090 \\
\hline Total places visited in A & .79 & $.41-1.51$ & .476 & .022 \\
\hline Total places visited in B & 2.24 & $.52-9.72$ & .281 & .061 & \\
\hline
\end{tabular}


Total places visited in C

Total places visited in D

(a) Not enough participants in this category to get results.

\section{Discussion}

The aim of the study was to explore how multiple various factors are associated with the perception of participation outside home for a sample of persons living with and without dementia in Switzerland. The results first show that the perception of participation is significantly associated with the number of places visited, but only for the persons living without dementia. Second, in the bivariate analysis, only a few factors are identified as significantly associated and even less are registering as significant when searching for a model, which might be underscoring the complexity of out-of-home participation as a construct. For the group of persons living with dementia, the results show that risks of falling and getting lost are a concern regarding the perception of participation outside home, and that the number of places visited does not seem to be associated. For the group of persons living without dementia being able to visit places in the $\mathrm{D}$ domain seem to be associated with a satisfying perception of participation outside home.

From our results, living with a dementia is a very significant factor that seems to change not only the number and types of places visited, but also how this relates to the perception of participation outside home. This is in line with former results of studies using ACT-OUT $(12,13)$, showing that having a dementia is likely to impact the way persons access, visit and use places outside home. These and former results question the assumption that the number of places visited is associated with the perception of participation outside home for persons living with dementia.

a) Association between the number of places visited and perceived participation

The results of the study show that for the comparison group, the number and diversity of places visited seems to be associated with perceived participation. It might mean that the more the older adults living without dementia visit a diversity of places the more they perceive their participation outside home to be satisfactory for them. However, for the group living with dementia, there is no such association. Persons having dementia tend to become more dependent on a significant other, and this would influence how, when and where they would go outside and what activities they would engage in (49). Out-of-home participation would be inter-influenced between the persons living with dementia and their significant others in a way that might be identified as co-occupations (50), offering another theoretical lens of looking at their participation. It might also be that those persons living with dementia resign form social activities and abandon places related to these activities. Similarly, as they reduce going there, the places might lose the meaning that formerly had been attributed to them (51); and as the disease progresses 
places would also lose their familiarity(12). Further research is needed to better understand the role that places visited play in participation outside home for persons living with dementia, including their meaning, interest, and support for engaging in co-occupations.

b) Exploration of associations between participation outside home and multiple variables

Our results on the exploration of multiple variables potentially being associated with perceived participation outside home, show that very few of those were significant, partially due to the small samples in this study. The education variable had to be taken out of the exploration as there was an insufficient number of participants in some categories.

Still, places, especially in domain D/places for recreational and physical activities, were associated with perceived participation for the comparison group, but not the group of persons living with dementia. However, for the latter group, places visited in domain $D$ was the closest of all types of places for being significant $(p=.062)$. Domain $D$ contains a range of places that are linked to physical activity, contact with nature, travelling and vacation, and the neighbourhood. Hence, it would be reasonable to expect an association between visiting these places, especially the neighbourhood (52) and perceived out-of-home participation. Physical activity has been shown to positively influence cognitive functioning, quality of sleep and perception of self, and prevent depression $(53,54)$. Contact with nature offers a restorative effect linked with greenery that increases positive emotions and reduces anger, and helps heighten awareness of self $(55,56)$. Older adults have also reported that travelling is important to them as it has shown to avoid disease, maintain high levels of physical and cognitive function, and especially engagement with life $(21,57,58)$. The neighbourhood has shown to be the support of social relations, engagement in life and participation outside home (9-11). Some of the places in domain D are very close to the person's home, like the garden around the house, or the neighbourhood, and others can be very far, like a summer house or a train station for travelling. In earlier studies, the abandonment rate of visiting the summer house or cottage was shown as being above $50 \%$ for the dementia group and above $20 \%$ for the comparison group $(12,13)$. As domain $D$ includes various places that each could have a potential association with perceived participation, further research is needed in determining how each place in this domain might be perceived by persons living with and without dementia. Also, more psychometric studies on ACT-OUT would provide insight into these issues of types of places and a better understanding of the perception of participation outside home.

It is interesting to note that no socio-demographic variable was identified as showing a significant association with perceived participation outside home in our results. As these variables are usually highlighted in research $(17,59)$ for having an impact on well-being and participation, the results of this study show a contradiction with earlier research. The results in this study point towards a more non-linear, complex and multifactorial understanding of perceived participation, as has also been stressed in earlier research (7). The lack of significant association in the results might also be due to the small sample size.

Two (getting lost and falling) of the four risks included seem to be significantly associated with perceived participation outside home, at least for the dementia group. As neither risk of getting lost nor falling were 
then reported significant in the search for a model (multivariate) for the dementia group, it seems that participants who perceive getting lost as a risk also perceive falling as one. Perceiving space outside home as fraught with risks (27) might be what is associated with perceived participation, instead of specific risks. There is a need to better understand how risks are perceived by persons living with dementia and their family $(38)$; and how they are socially constructed in the local context $(28,37)$ in terms of social biais. Furthermore, although risks are often seen as having a restricting association with participation outside home, especially for persons living with dementia (28), some authors have argued that risks could be perceived as positive, offering challenges that will "add a spark" to life (31). Supporting a more positive representation of risks when related to out-of-home participation of older adults $(60,61)$, and especially those living with dementia, might also increase their opportunities to maintain visiting important and meaningful places; and incidentally address the stigmatisation of having a dementia in our society by recognising continued citizenship $(62,63)$.

\section{Study limitations}

The small sample size is a limitation of this study that need attention. Although 70 participants were included in the full sample, the factor of living with a dementia was shown to be significant in the analysis, supporting the idea of conducting the exploration of multiple factors separately for both groups. As the separate group were made of small samples, we first used bivariate analysis before looking for a model, to mitigate that effect. Looking for a model with small samples is tentative, as the number of factors and steps in the scales adds to the number of freedom degrees, and might make the results less reliable. Therefore, our results need to be considered within this limitation. Still, this exploratory study gives insights into further explorations of participation outside home for persons living with dementia.

Another limitation of this study is that we did not collect data about each participant amenities and transportation availability, which could potentially have been included in the factors under exploration. It is uncertain how the absence of these variables might have changed our results. In addition to sociodemographic determinants, perceived participation outside home is dependent on individual preferences, making this construct very complex to apprehend. There is a need to better specify the impact of sociodemographic factors on participation outside home in future longitudinal studies for persons living with dementia.

Collecting data with persons living with dementia through the use of face-to face interviews raises the question of the reliability of discourse $(64,65)$, especially to rely on episodic memory to narrate autobiographical facts. However, in this study, the interviews concerned perception of out-of-home participation, the perception of risks and socio-demographic variables.

\section{Conclusion}

We found that participation outside home is associated with the number of places visited, but only for the group of persons living without known impairments. Our exploration of multiple factors and search for a 
model, highlights the complexity of participation outside home as a construct. Visiting multiple and various types of places seem to play a role in participation outside home but would need to be further investigated. Longitudinal studies are needed to investigate causal relationships between the different associated factors and to evaluate the best way of supporting participation outside home for persons living with dementia.

\section{Declarations}

\section{- Ethics approval and consent to participate}

Ethical approval was sought and obtained from the Ethics committee of canton de Vaud (CER-VD) under the reference number 452/15. All methods were performed in accordance with the relevant guidelines and regulations in place at the time of the data collection. Written and verbal informed consent was obtained from each participant prior to data collection and assent to participate was monitored during the data collection.

\section{- Consent for publication}

Consent for publication - Not applicable.

\section{- Availability of data and materials}

The datasets generated and/or analysed during the current study are not publicly available due to ongoing international secondary analysis but are available from the corresponding author on reasonable request.

The data (from Switzerland) used in this study has also been shared within the ACT-OUT development project under a data sharing convention and funding from the Kampraad Family Foundation. The larger dataset comprises data from 4 countries (Sweden, Switzerland, UK and Canada) and is currently being analysed in comparison to each country. Access to the combined dataset may also be granted upon reasonable request.

\section{- Competing interests}

No potential conflict of interest was reported by the authors.

\section{- Funding}

The work in Switzerland was supported by the Swiss Alzheimer Association (https://www.alzheimerschweiz.ch/fr/les-demences/article/projets-de-recherche-soutenus/); and the Swiss Occupational Therapy Association. The work in Sweden was supported by the Swedish Research Council for Health, Working Life and Welfare (FORTE/ 2013-2104) and the Kamprad Family Foundation (20180057). 


\section{- Authors' contributions}

I.M. and L.N. wrote the main manuscript text, collected the data and performed the analysis with supervision from A.B. and C.M. I.M. A.B., S.G. and C.M. prepared the tables and figures. N.K. mainly contributed to the introduction and discussion, and to the risk perception inclusion in the study. All authors reviewed the manuscript.

\section{- Acknowledgements}

We thank all study participants for offering their time and for letting us hear their voices, while they were living in a difficult situation. We also thank Delphine Dumoulin for her contribution in collecting the data. Special thanks to the CACTUS research group at Karolinska Institutet for continued support and critical discussions. We want to have special thanks to all neuropsychologists and physicians who believed in this project and referred the participants living with dementia to us.

\section{References}

1. Cerejeira J, Lagarto L, Mukaetova-Ladinska EB. Behavioral and Psychological Symptoms of Dementia. Front Neurol [Internet]. 2012 [cited 2020 Sep 11];3. Available from: http://journal.frontiersin.org/article/10.3389/fneur.2012.00073/abstract

2. Hodges JR, Patterson K. Semantic dementia: a unique clinicopathological syndrome. Lancet Neurol. 2007 Nov;6(11):1004-14.

3. Levine TF, Allison SL, Stojanovic M, Fagan AM, Morris JC, Head D. Spatial navigation ability predicts progression of dementia symptomatology. Alzheimers Dement. 2020 Mar;16(3):491-500.

4. Tucker-Drob EM. Cognitive Aging and Dementia: A Life-Span Perspective. Annu Rev Dev Psychol. 2019 Dec 24;1(1):177-96.

5. Nay R, Bauer M, Fetherstonhaugh D, Moyle W, Tarzia L, McAuliffe L. Social participation and family carers of people living with dementia in Australia. Health Soc Care Community. 2015 Sep;23(5):5508.

6. Sugarhood P, Eakin P, Summerfield-Mann L. Participation in advanced age: enacting values, an adaptive process. Ageing Soc. 2017 Sep;37(8):1654-80.

7. Argyle $E$, Dening T, Bartlett P. Space, the final frontier: outdoor access for people living with dementia. Aging Ment Health. 2017 Oct 3;21(10):1005-6.

8. Duggan S, Blackman T, Martyr A, Van Schaik P. The impact of early dementia on outdoor life. Dementia. 2008 May 1;7(2):191-204.

9. Clark A, Campbell S, Keady J, Kullberg A, Manji K, Rummery K, et al. Neighbourhoods as relational places for people living with dementia. Soc Sci Med. 2020 May;252:112927.

10. Odzakovic E, Hellström I, Ward R, Kullberg A. 'Overjoyed that I can go outside': Using walking interviews to learn about the lived experience and meaning of neighbourhood for people living with 
dementia. Dementia. 2018;19(7):2199-219.

11. Ward R, Clark A, Campbell S, Graham B, Kullberg A, Manji K, et al. The lived neighborhood: understanding how people with dementia engage with their local environment. Int Psychogeriatr. 2017 May 2;1-14.

12. Margot-Cattin I, Ludwig C, Kühne N, Eriksson G, Berchtold A, Nygard L, et al. Visiting Out-of-Home Places when Living with Dementia. Can J Occup Ther. 2021 Mar 22;88(2):131-41.

13. Gaber SN, Nygård L, Brorsson A, Kottorp A, Malinowsky C. Everyday technologies and public space participation among people with and without dementia. Can J Occup Ther. 2019 May 29;000841741983776.

14. Blackman T, Mitchell, L, Burton, E, Jenks, M, Parsons, M, Raman, S, et al. The Accessibility of Public Spaces for People with Dementia: a new priority for the open city. Disabil Soc. 2003;18(3):357-71.

15. Brorsson A, Öhman A, Lundberg S, Nygård L. Accessibility in public space as perceived by people with Alzheimer's disease. Dementia. 2011;10(4):587-602.

16. Malinowsky C, Olofsson A, Nyman A, Lund ML. Patterns of participation: Facilitating and hindering aspects related to places for activities outside the home after stroke. Scand J Occup Ther. 2019 Sep 28;1-9.

17. Stubbs B, Eggermont L, Soundy A, Probst M, Vandenbulcke M, Vancampfort D. What are the factors associated with physical activity (PA) participation in community dwelling adults with dementia? A systematic review of PA correlates. Arch Gerontol Geriatr. 2014 Sep;59(2):195-203.

18. Gilmour H. Living alone with dementia: risk and the professional role. Nurs Older People. 2004;16(9):20-4.

19. Innes A, Morgan D, Farmer J. Remote and rural dementia care: policy, research and practice. 2020.

20. Battista GA, Manaugh K. Stores and mores: Toward socializing walkability. J Transp Geogr. 2018 Feb;67:53-60.

21. Shergold I. Taking part in activities, an exploration of the role of discretionary travel in older people's wellbeing. J Transp Health. 2019 Mar;12:195-205.

22. Gaber SN, Nygård L, Brorsson A, Kottorp A, Charlesworth G, Wallcook S, et al. Social Participation in Relation to Technology Use and Social Deprivation: A Mixed Methods Study Among Older People with and without Dementia. Int J Environ Res Public Health. 2020 Jun 5;17(11):4022.

23. Yeom HA, Fleury J, Keller C. Risk Factors for Mobility Limitation in Community-Dwelling Older Adults: A Social Ecological Perspective. Geriatr Nur (Lond). 2008 Mar;29(2):133-40.

24. Choi M, Lohman MC, Mezuk B. Trajectories of cognitive decline by driving mobility: evidence from the Health and Retirement Study: Driving and cognitive trajectories. Int J Geriatr Psychiatry. 2014 May;29(5):447-53.

25. Bailey C, Clarke CL, Gibb C, Haining S, Wilkinson H, Tiplady S. Risky and resilient life with dementia: review of and reflections on the literature. Health Risk Soc. 2013 Aug;15(5):390-401. 
26. Clarke CL, editor. Risk assessment and management for living well with dementia. London; Philadelphia: Jessica Kingsley Publishers; 2011. 128 p. (Bradford Dementia Group good practice guides).

27. Neubauer N, Liu L. Development and validation of a conceptual model and strategy adoption guidelines for persons with dementia at risk of getting lost. Dementia. 2020 Jan 22;147130121989835.

28. Berry B, Apesoa-Varano EC, Gomez Y. How family members manage risk around functional decline: The autonomy management process in households facing dementia. Soc Sci Med. 2015 Apr;130:107-14.

29. Lloyd BT, Stirling C. The will to mobility: life-space satisfaction and distress in people with dementia who live alone. Ageing Soc. 2015 Oct;35(9):1801-20.

30. Lupton D, editor. Risk and sociocultural theory: new directions and perspectives. Cambridge; New York: Cambridge University Press; 1999. 191 p.

31. Waring A. Constructive risk in the care of the older adult: a concept analysis. Br J Nurs. $2000 \mathrm{Jul}$ 27:9(14):916-24.

32. Alaszewski A, editor. Managing risk in community practice: nursing, risk and decision making. Edinburgh: Baillière Tindall; 2000. 190 p.

33. Fischhoff B, Kadvany JD. Risk: a very short introduction. Oxford; New York: Oxford University Press; 2011. 162 p. (Very short introductions).

34. Emrich-Mills L, Puthusseryppady V, Hornberger M. Effectiveness of Interventions for Preventing People With Dementia Exiting or Getting Lost. C. Heyn P, editor. The Gerontologist [Internet]. 2019 Oct 31 [cited 2020 Aug 17]; Available from: https://academic.oup.com/gerontologist/advancearticle/doi/10.1093/geront/gnz133/5610511

35. Pai M-C, Lee C-C.The Incidence and Recurrence of Getting Lost in Community-Dwelling People with Alzheimer's Disease: A Two and a Half-Year Follow-Up. Sokolov AN, editor. PLOS ONE. 2016 May 16;11(5):e0155480.

36. Nyman SR, Ballinger C, Phillips JE, Newton R. Characteristics of outdoor falls among older people: a qualitative study. BMC Geriatr [Internet]. 2013 Dec [cited 2020 Oct 26];13(1). Available from: http://bmcgeriatr.biomedcentral.com/articles/10.1186/1471-2318-13-125

37. Bantry White E, Montgomery P. Supporting people with dementia to walkabout safely outdoors: development of a structured model of assessment. Health Soc Care Community. 2016;24(4):473-84.

38. Droz-Mendelzweig M, Chappuis M, Vuilleumier D, Kuhne N, Piguet C, Bedin MG.Le risque chez les personnes âgées à domicile: un objet relationnel.Rev Médicale Suisse. 2014;1835-7.

39. Sandberg L, Rosenberg L, Sandman P-O, Borell L. Risks in situations that are experienced as unfamiliar and confusing - the perspective of persons with dementia. Dementia. 2017 May;16(4):471-85.

40. Margot-Cattin I, Kuhne N, Kottorp A, Cutchin M, Öhman A, Nygard L. Development of a Questionnaire to Evaluate Out-of-Home Participation for People With Dementia. Am J Occup Ther. 2019;73(1):1- 
10.

41. Chaudhury H, Mahal T, Seetharaman K, Nygaard HB. Community participation in activities and places among older adults with and without dementia. Dementia. 2020 May 29;147130122092723.

42. Pezalla AE, Pettigrew J, Miller-Day M. Researching the researcher-as-instrument: an exercise in interviewer self-reflexivity. Qual Res. 2012 Apr;12(2):165-85.

43. Nasreddine ZS, Phillips NA, Bédirian V, Charbonneau S, Whitehead V, Collin I, et al. The Montreal Cognitive Assessment, MoCA: a brief screening tool for mild cognitive impairment. J Am Geriatr Soc. 2005;53(4):695-9.

44. Dewing J. From Ritual to Relationship. Dementia. 2002 Jun 1;1(2):157-71.

45. Dewing J. Participatory research: A method for process consent with persons who have dementia. Dementia. 2007 Feb 1;6(1):11-25.

46. Mckeown J, Clarke A, Ingleton C, Repper J. Actively involving people with dementia in qualitative research. J Clin Nurs. 2010;19(13-14):1935-43.

47. UNESCO Institute for Statistics. International Standard Classification of Education (ISCED 2011). Montréal, QC, Canada: UNESC Institute for Statistics; 2012 p. 88.

48. Cohen J. Statistical power analysis for the behavioral sciences (2nd ed).Hillsdale, NJ: Erlbaum; 1988. $474 \mathrm{p}$.

49. Couture N, Éthier S. L'impact de la maladie d'Alzheimer sur la participation sociale des personnes qui en sont atteintes et leurs proches.Une fatalité? Loisir Société Soc Leis. 2020 Jan 2;43(1):16-35.

50. Ono K, Kanayama Y. Views on Co-occupation between Elderly Persons with Dementia and Family. J Gerontol Geriatr Res [Internet]. 2014 [cited 2020 Nov 2];03(05). Available from:

https://www.omicsgroup.org/journals/views-on-co-occupation-between-elderly-persons-withdementia-and-family-2167-7182.1000185.php?aid=37040

51. Fang ML, Woolrych R, Sixsmith J, Canham S, Battersby L, Sixsmith A. Place-making with older persons: Establishing sense-of-place through participatory community mapping workshops. Soc Sci Med. 2016 Nov;168:223-9.

52. Ward R, Rummery K, Odzakovic E, Manji K, Kullberg A, Keady J, et al. Beyond the shrinking world: dementia, localisation and neighbourhood. Ageing Soc. 2021 Mar 22;1-22.

53. Cedervall Y, Torres S, Åberg AC. Maintaining well-being and selfhood through physical activity: experiences of people with mild Alzheimer's disease. Aging Ment Health. 2014 Sep 30;1-10.

54. Winchester J, Dick MB, Gillen D, Reed B, Miller B, Tinklenberg J, et al. Walking stabilizes cognitive functioning in Alzheimer's disease (AD) across one year. Arch Gerontol Geriatr. 2013 Jan;56(1):96103.

55. Gibson G, Chalfont GE, Clarke PD, Torrington JM, Sixsmith AJ. Housing and Connection to Nature for People with Dementia. J Hous Elder. 2007 Dec 17;21(1-2):55-72.

56. Whear R, Coon JT, Bethel A, Abbott R, Stein K, Garside R. What Is the Impact of Using Outdoor Spaces Such as Gardens on the Physical and Mental Well-Being of Those With Dementia? A Systematic 
Review of Quantitative and Qualitative Evidence. J Am Med Dir Assoc. 2014 Oct;15(10):697-705.

57. Ahn Y, Janke MC. Motivations and Benefits of the Travel Experiences of Older Adults. Educ Gerontol. 2011 Aug;37(8):653-73.

58. Cerin E, Nathan A, van Cauwenberg J, Barnett DW, Barnett A. The neighbourhood physical environment and active travel in older adults: a systematic review and meta-analysis. Int J Behav Nutr Phys Act [Internet]. 2017 Dec [cited 2020 Dec 4];14(1).Available from: http://ijbnpa.biomedcentral.com/articles/10.1186/s12966-017-0471-5

59. Martyr A, Nelis SM, Quinn C, Wu Y-T, Lamont RA, Henderson C, et al.Living well with dementia: a systematic review and correlational meta-analysis of factors associated with quality of life, wellbeing and life satisfaction in people with dementia. Psychol Med. 2018 Oct;48(13):2130-9.

60. Mapes N. Think outside: positive risk-taking with people living with dementia. Work Older People. 2017 Sep 11;21(3):157-66.

61. Stevenson M, McDowell ME, Taylor BJ. Concepts for communication about risk in dementia care: A review of the literature. Dementia. 2018 Apr;17(3):359-90.

62. Brannelly T. Sustaining citizenship: People with dementia and the phenomenon of social death. Nurs Ethics. 2011 Sep;18(5):662-71.

63. Nguyen T, Li X. Understanding public-stigma and self-stigma in the context of dementia: A systematic review of the global literature. Dementia. 2020 Feb;19(2):148-81.

64. Dijkstra K, Bourgeois MS, Allen RS, Burgio LD. Conversational coherence: discourse analysis of older adults with and without dementia. J Neurolinguistics. 2004 Jul;17(4):263-83.

65. Seixas-Lima B, Murphy K, Troyer AK, Levine B, Graham NL, Leonard C, et al. Episodic memory decline is associated with deficits in coherence of discourse. Cogn Neuropsychol. 2020 Nov 16;37(78):511-22.

\section{Figures}




\section{Number of visited places by Participation categories and group (means and SD)}

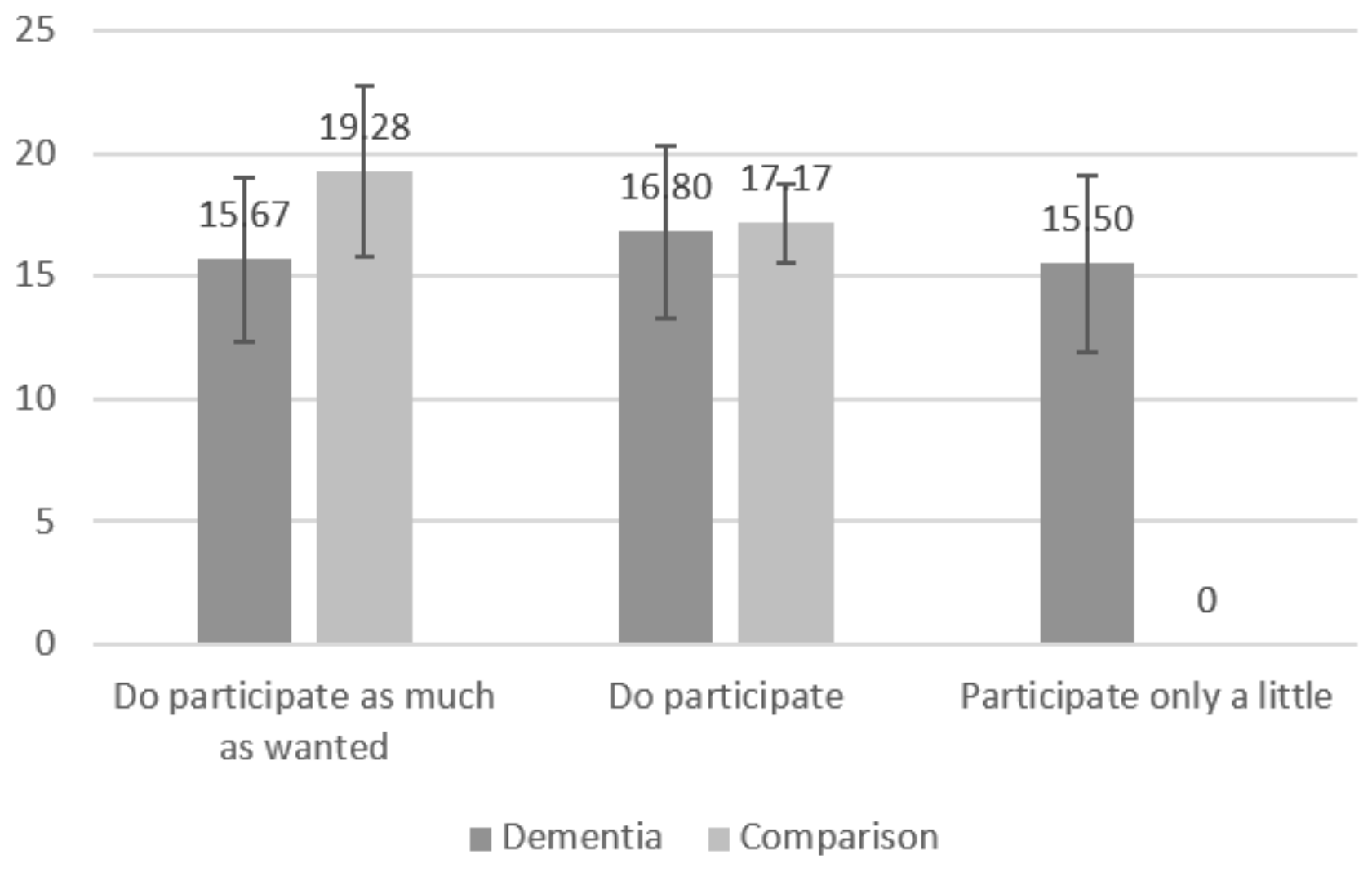

Figure 1

Mean number of places visited per level of perceived participation and per group. 